\title{
Introducción de la Metodología Design Thinking en estudiantes del Grado de Finanzas y Contabilidad
}

\section{Begoña Cabanés-Cacho ${ }^{a}$, Susana Rubio-Arraez}

aFacultad de Economía y Empresa, Universidad de Zaragoza, bcabanes@unizar.es, 'bnstituto Universitario de Ingeniería de Alimentos para el Desarrollo. Universitat Politècnica de València, suruar@upvnet.upv.es

\begin{abstract}
Design Thinking is a full methodology that allows us to obtain innovative ideas to solve real-world problems that people face in their lives. The aim of this study is the implementation of this methodology in the subject of Strategic Planning in the Degree of Finance and Accounting in the University of Zaragoza, in order to introduce it and develop at future editions. Students will be evaluate using a questionnaire, once the last phase of the Design Thinking is completed, to determine if objectives have been achieved and in which degree they have influenced to the students. In conclusion, through the Design Thinking methodology, students are expected to be able to solve problems and challenges generated in business environment. In this way, among other things, the transversal competences of the subject such as teamwork, collaboration, and development of a critical attitude in any financial environment, are also encouraged.
\end{abstract}

Keywords: design thinking, brainstorming, finance, strategic planning, educational innovation.

\section{Resumen}

El Design Thinking es una metodología muy completa que nos permite obtener ideas innovadoras que solucionan problemas reales e importantes para las personas. El objetivo de este estudio es la implementación de esta metodología en la asignatura Planificación Estratégica del Grado de Finanzas y Contabilidad de la Universidad de Zaragoza, a modo de introducción y posterior desarrollo en ediciones sucesivas. Se evaluará a los alumnos, una vez finalizada la última fase del Design Thinking, por medio de un cuestionario, para determinar si los objetivos se han alcanzado y en qué grado han influido en los alumnos. En conclusión, a través de la metodología Design Thinking, se espera que los alumnos sean capaces de solucionar problemas y retos que se generaran en el ambiente empresarial. De esta manera, se incentivan a su vez, las competencias transversales propias de la asignatura como el trabajo en equipo, colaboración, desarrollo de una actitud crítica en cualquier entorno financiero entre otras. 
Palabras clave: design thinking, tormenta de ideas, finanzas, planificación estratégica, innovación educativa.

\section{Introducción}

\subsection{Grado de Finanzas y Contabilidad y el futuro laboral}

En la actualidad, estamos formando a los alumnos para un mundo globalizado y con un gran desarrollo digital, pero que sigue en constante evolución y cambio en el que están apareciendo nuevas tecnologías como Internet de las Cosas (IoT), realidad aumentada y virtual, Cloud, Big Data, Blockchain, digitalización, entre otras. Esto va a repercutir en el medio plazo en el mercado laboral. Los medios de comunicación ya se están haciendo eco de los últimos estudios realizados sobre como la tecnología va a afectar a los puestos de trabajo actuales. Según el informe The future of employment "el $47 \%$ de los empleos actuales desaparecerán en los próximos 25 años" . En esta línea, el informe realizado por Manpower Group cifra en un 30\% los empleos que desaparecerán con la transformación tecnológica y prevé que el $65 \%$ de los trabajos experimentara cambios ${ }^{2}$. Por otro lado, el Foro Económico Mundial (FEM) en su estudio "El Futuro del Trabajo 2018" ${ }^{3}$ nos anticipa a que los trabajadores deberán actualizarse y formarse en esas áreas para poder adaptarse, es decir, tendrán que desarrollar sus capacidades y conocimientos tecnológicos. Pero eso no será suficiente para el modelo productivo al que nos dirigimos, intensivo en conocimiento y con un mayor valor añadido. Con lo cual no va a ser suficiente desarrollar las competencias tecnológicas, sino que nos describen que capacidades y competencias van a demandarse en el futuro, como son:

- creatividad, originalidad e iniciativa,

- pensamiento crítico y capacidad analítica,

- innovación,

- habilidades de trabajo en equipo,

- resolución de problemas complejos,

- actualización permanente

Los cambios que se avecinan no somos capaces de imaginarlos, quizás tengamos que aprender a relacionarnos y trabajar con robots. En el pasado Internet ayudo a desarrollar nuevos modelos de negocio como Amazon, Airbnb, Cabify o Uber, entre otros. La

\footnotetext{
1 “El 47\% de los empleos actuales desaparecerán en los próximos 25 años”, 22/08/2018 Periódico digital Huffington Post https://www.huffingtonpost.es/fernando-bruccoleri/el-47-de-los-empleos-actuales-desapareceran-en-los-proximos-25anos_a_23503110/

2 “Un 30\% de los empleos desaparecerá con la transformación tecnológica, según Manpower Group”, 03/02/2019 a través de la web https://www.eleconomista.es/empresas-finanzas/noticias/9676304/02/19/Manpowergroup-preve-que-un-30-de-empleosdesapareceran-con-la-transformacion-tecnologica.html

3 “Más de la mitad de los empleos actuales serán reemplazados por robots en 2025” 19/09/2018 Periódico digital ABC, articulo de Marta Hurtado (EFE) https:/www.abc.es/tecnologia/informatica/soluciones/abci-mas-mitad-empleos-actuales-seranreemplazados-robots-2025-201809190204_noticia.html
} 
dificultad del sistema educativo está en la velocidad con la que se suceden los cambios tecnológicos, que hacen que se vaya quedando rezagado. Estamos hablando que deberíamos preparar a nuestros estudiantes para cambios disruptivos. En el caso de estos alumnos del Grado de Finanzas y Contabilidad van a trabajar en un sector que se va a verse fuertemente influenciado.

Por eso desde la asignatura Planificación Estratégica de la empresa, del tercer curso del Grado de Finanzas y Contabilidad de la Facultad de Economía y Empresa de la Universidad de Zaragoza en el curso 2019-20, se plantea la implementación de la metodología del Design Thinking para ayudar a los alumnos a desarrollar las competencias demandadas cada vez más como son la creatividad, pensamiento crítico y resolución de problemas complejos.

\subsection{Qué es Design Thinking}

Design Thinking es una herramienta que nos permite la resolución de problemas desde una nueva orientación poniendo al usuario en el centro del eje. Esta metodología proviene de aplicar el pensamiento de los especialistas en diseño de productos hacia otros campos o áreas.

De esta forma al crear una idea, se piensa en el usuario que va a llevarla a término, por ende, el éxito de dicha idea será mayor en cuanto más se acerque a las necesidades de los usuarios. En este sentido, a mayor investigación sobre el área de desarrollo, nos conducirá a una mayor innovación. No obstante, Design Thinking vincula el pensamiento creativo con el analítico, con el fin de fomentar la motivación, la empatía y el aspecto lúdico, para elaborar ideas ajustadas a las necesidades reales del usuario (Brown, 2008, 2009,2010).

Según Brown (2008) "Design thinking no es un arte, ni una ciencia ni una religión es la capacidad del pensamiento integrador". El proceso consta de cinco etapas: Empatía, Definición, Idea, Prototipo y Test. Todas las fases van unidas de una forma coherente y práctica donde se parte de identificar un problema, seguido del desarrollo e investigación de nuevas ideas, se observan los retos, se detectan las necesidades y, posteriormente, se produce el desarrollo de soluciones.

Sin embargo, la resolución de los problemas del usuario y la satisfacción de sus necesidades debe aunar en tres aspectos lógicos: personal, comercial y tecnológicamente viable. Por otro lado, el Design Thinking requiere dinámicas grupales y equipos multidisciplinares, realizar prototipos y testear los productos antes de ser lanzados para visualizar la idea y vislumbrar los resultados.

Esta estructura se materializa a través de las cinco etapas del proceso de Design Thinking: La primera etapa (Empatizar) consiste en obtener una comprensión empática del problema a resolver. Esto implica consultar al usuario para obtener más información sobre el área de su interés para entender sus experiencias y motivaciones. La empatía es crucial para un proceso de diseño centrado en el ser humano, junto con el pensamiento del diseño permiten a los creadores dejar de lado sus propias suposiciones con el fin de obtener una visión de 
los usuarios y sus necesidades. En esta etapa se recopila una cantidad sustancial de información para utilizar durante la siguiente etapa y la mayor comprensión posible de las necesidades y problemas del usuario que subyacen en el desarrollo del producto (Dijksterhuis \& Silvius, 2017; Mosely, Wright \& Wrigley, 2018).

Durante la segunda etapa (Definir), recopilada la información, se analizan las observaciones y se sintetizan para concretar los principales problemas identificados. El objetivo es solucionar los problemas con la menor dificultad. En la tercera etapa (Idea), se formulan preguntas que pueden ayudar a generar ideas (Brainstorm), preguntándonos qué tipo de acción se puede realizar para producir un beneficio en el sector, humanizando la idea (Brown, 2010; Benson, \& Dresdow, 2014).

En la cuarta etapa se da el Prototipado de un diseño, en una versión económica, a escala reducida del producto para poder investigar las soluciones a los problemas generados en la etapa anterior. Los prototipos pueden ser compartidos y probados dentro del propio equipo, en otros departamentos, o en un pequeño grupo de personas fuera del equipo de diseño. Esta es una fase experimental y el objetivo es identificar la mejor solución posible para cada uno de los problemas observados durante las primeras tres etapas (Brown, 2009; Glen, Suciu, Baughn \& Anson, 2015; Wrigley \& Straker, 2017; Mosely, Wright \& Wrigley, 2018).

En la última fase (Test) se investigan y se mejoran las ideas, se pueden reexaminar o incluso rechazar en base a las experiencias de los usuarios. De esta forma se tiene una visión más clara de cómo los usuarios reales se comportan, piensan y sienten cuando interactúan con el producto final. Los diseñadores o evaluadores prueban rigurosamente el producto completo utilizando las mejores soluciones identificadas durante la fase de prototipado (Brown, 2010; Mosely, Wright \& Wrigley, 2018).

En los apartados siguientes vamos a explicar cómo se va a materializar la implementación de la metodología descrita, definiendo en primer lugar los objetivos que perseguimos.

\section{Objetivos}

A la vista del entorno que se van a encontrar los alumnos en su incorporación al mundo laboral, se decide utilizar la metodología Design Thinking en el trabajo que tienen que elaborar en la asignatura. Al incorporar esta metodología les ayudara a desarrollar las competencias que les pedirán en el mundo laboral. Estableciendo una sinergia positiva con las competencias transversales y específicas que deben adquirir en la asignatura. Las competencias específicas de la asignatura son:

- Comprender la naturaleza de la empresa e instituciones, su relación con el entorno económico, jurídico, social y medioambiental y la incidencia del mismo sobre las áreas financiera y contable de las organizaciones.

- Entender los contextos en los que las finanzas y la contabilidad se relacionan individual y conjuntamente con otras áreas funcionales de las organizaciones.

- Participar en el asesoramiento a empresas, instituciones e inversores en la gestión y administración de los recursos financieros desde un enfoque integral. 
Y las Competencias transversales son:

- capacidad para la resolución de problemas,

- capacidad para la toma de decisiones,

- desarrollar actitudes colaborativas y de trabajo en equipos multidisciplinares o multiculturales, así, como desarrollar una actitud crítica para el debate,

- capacidad para adaptarse a entornos dinámicos con espíritu creativo.

El objetivo general es ayudar a los alumnos a desarrollar las competencias más demandadas en el mercado, ya que están directamente relacionadas con las competencias recogidas en la guía docente de la asignatura tal y como se recoge en la Tabla 1.

Tabla 1. Relación entre las competencias que exige el mercado laboral y las competencias transversales de la asignatura.

\begin{tabular}{|c|c|c|c|c|c|}
\hline & & \multicolumn{4}{|c|}{ Competencias transversales de la asignatura } \\
\hline & & $\begin{array}{c}\text { Capacidad para la } \\
\text { resolución de } \\
\text { problemas }\end{array}$ & $\begin{array}{c}\text { Capacidad para la } \\
\text { toma de } \\
\text { decisiones }\end{array}$ & $\begin{array}{c}\text { Desarrollar actitudes } \\
\text { colaborativas y de trabajo en } \\
\text { equipos multidisciplinares o } \\
\text { multiculturales, asi, como } \\
\text { desarrollar una actitud crítica } \\
\text { para el debate. }\end{array}$ & $\begin{array}{l}\text { Capacidad para } \\
\text { adaptarse a } \\
\text { entornos } \\
\text { dinámicos con } \\
\text { espiritu creativo. }\end{array}$ \\
\hline \multirow{6}{*}{$\begin{array}{c}\text { Competencias } \\
\text { que exige el } \\
\text { mercado } \\
\text { laboral }\end{array}$} & $\begin{array}{l}\text { Creatividad, originalidad e } \\
\text { iniciativa }\end{array}$ & & $\mathrm{x}$ & & $\mathrm{x}$ \\
\hline & $\begin{array}{l}\text { Pensamiento critico y } \\
\text { capacidad analitica }\end{array}$ & & $\mathrm{x}$ & $\mathrm{x}$ & \\
\hline & Innovación & & $\mathrm{x}$ & & \\
\hline & $\begin{array}{l}\text { Habilidades de trabajo en } \\
\text { equipo }\end{array}$ & & & $\mathrm{x}$ & $\mathrm{x}$ \\
\hline & $\begin{array}{l}\text { Resolución de problemas } \\
\text { complejos }\end{array}$ & $\mathrm{x}$ & $\mathrm{x}$ & & \\
\hline & Actualización permanente & & & $\mathrm{x}$ & $\mathrm{x}$ \\
\hline
\end{tabular}

Fuente: Elaboración propia

Los objetivos específicos tratan de que el alumno sea consciente de que nivel tiene en cada competencia a través de un cuestionario, que para determinados objetivos se utilizaran varios ítems. El alumno a través del cuestionario nos indicara si se ha mejorado su competencia al realizar este trabajo.

Los objetivos específicos son:

- Mejorar la capacidad creativa del alumno

- Desarrollar el pensamiento crítico y capacidad analítica del alumno

- Conocer su perfil innovador, preguntando sobre si se siente cómodo saliendo de la zona de confort, si detecta soluciones más disruptivas que las expuestas por sus compañeros, si asume riesgos, ...

- Desarrollar la habilidad de trabajo en equipo.

- Mejorar la capacidad de resolución de problemas complejos del alumno 
- Conocer su actitud hacia la formación a lo largo de la vida o actualización permanente. Clasificándose en un rol activo o pasivo ante la formación continúa.

Por tanto, los objetivos específicos se valoraran a través de un cuestionario donde los alumnos hagan una valoración de si han mejorado o no sus competencias.

\section{Desarrollo de la innovación}

Los alumnos tienen que realizar un trabajo sobre una empresa que eligen previamente con el visto bueno del profesor. Deben analizar la situación de la empresa, a través de un análisis interno y externo de la misma basándose en la teoría vista en clase para finalmente realizar un plan estratégico. En esta última parte es donde entra la metodología Design Thinking. Se fijaran 6 días de clase para abordar este trabajo. El primer día tras un trabajo previo de investigación deberán completar el Business Model Canvas de la empresa, o también llamado Canvas de modelo de negocio o Lienzo de modelo de negocio. Donde se recogen los aspectos clave de una empresa: socios, actividades, recursos, propuesta de valor, relaciones con los clientes, canales, segmentos de clientes, estructura de costes y líneas de ingresos.

En el segundo día realizaremos la primera fase del Design Thinking, empatía, seleccionando una de las herramientas como son el mapa de empatía, o el Role play, que nos permitirá recopilar diferentes perspectivas para empezar a entender cuál es el problema a resolver. Como la información que tenemos de la empresa es limitada, el profesor planteara el problema a resolver en función de la actividad y características de la empresa seleccionada. Los problemas que se plantearan serán como la empresa puede incorporar los Objetivos de Desarrollo Sostenible (ODS), las nuevas tecnologías como Internet de las Cosas (IoT), realidad aumentada y virtual, Cloud, Big Data, Blockchain, digitalización, impresión 3D, entre otros retos que existen actualmente.

En el tercer día, realizaremos la fase de definición basándonos en la información recogida el día anterior. Tienen que encontrar cual es problema que engloba la mayoría de las incidencias detectadas.

Durante el cuarto día, deben buscar diferentes soluciones al problema. Se trata de una jornada similar a la lluvia de ideas o brainstorming, donde lo importante es recopilar el mayor número de ideas para luego hacer diferentes cribas hasta quedarte con una.

En el quinto día deben diseñar de forma económica y a escala reducida cual es la solución al problema. Es decir, desarrollar la idea seleccionada el día anterior, creando si es posible un prototipo.

Para superar la última fase, el test, donde los alumnos deberían presentar a la empresa el prototipo para detectar fallos y mejorar el producto antes de ponerlo en marcha. Como esto va a ser muy complicado, aprovecharemos la sesión donde los alumnos presentan su trabajo a los compañeros y al profesor para que el resto de los alumnos realice una crítica constructiva explicando si han resuelto el problema que se planteaban o no y áreas de mejora. 


\section{Resultados}

Los resultados podremos valorarlos una vez se desarrolle esta metodología en la asignatura de Planificación Estratégica de la empresa durante el curso 2019-20, durante los meses de septiembre de 2019 a enero de 2020. Después de la última fase del Design Thinking y antes de acabar la asignatura, en enero de 2020, se pasara un cuestionario a los alumnos para cuantificar el impacto de esta metodología sobre ellos y valorar si se han alcanzado los objetivos planteados.

\section{Conclusiones}

A través del Design Thinking se espera ayudar a los alumnos a que piensen de otra manera para solucionar problemas y retos que se dan en las empresas. Y además, les permita desarrollar las competencias transversales que necesitaran en el futuro como son: capacidad para la resolución de problemas y la toma de decisiones; desarrollar actitudes colaborativas y de trabajo en equipos multidisciplinares o multiculturales, así, como desarrollar una actitud crítica para el debate; $\mathrm{y}$, capacidad para adaptarse a entornos dinámicos con espíritu creativo.

\section{Referencias}

BROWN, T. (2008). “Design thinking”. Harvard Business Review, 86, 9, 62-72.

BROWN, T. (2009). Change by Design: How Design Thinking Transforms Organizations and Inspires Innovation. New York: HarperBusiness.

BROWN, T., WYATT, J. (2010). “Design Thinking for Social Innovation”. Stanford Social Innovation Review, 31-35.

MOSELY, G., WRIGHT, N., WRIGLEY, C. (2018). "Facilitating design thinking: A comparison of design expertise”. Thinking Skills and Creativity, 27, 177-189.

BENSON, J., DRESDOW, S. (2014). "Design thinking: A fresh approach for transformative assessment practice". Journal of Management Education, 38(3), 436-461.

DIJKSTERHUIS, E., SILVIUS, G. (2017). “The design thinking approach to projects". The Journal of Modern Project Management, 4(3), 33-41.

WRIGLEY, C., STRAKER, K. (2017). "Design thinking pedagogy: The educational design ladder". Innovations in Education and Teaching International, 54(4), 374-385.

GLEN, R., SUCIU, C., BAUGHN, C. C., ANSON, R. (2015). “Teaching design thinking in business schools”. The International Journal of Management Education, 13,182-192. 